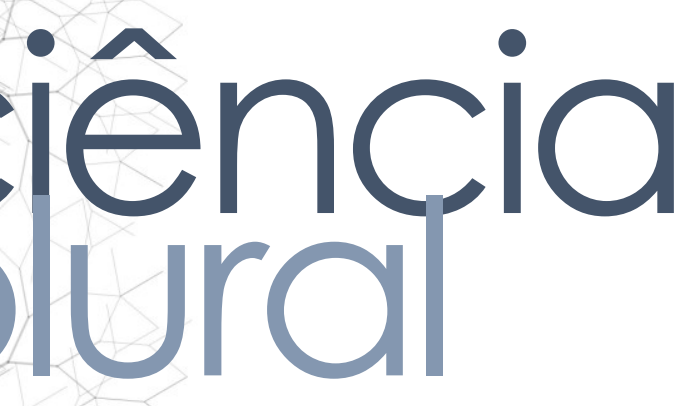

\title{
PERFIL DAS PESSOAS EM SITUAÇÃO DE FRAGILIDADE SOCIAL ATENDIDAS PELO "CONSULTÓRIO NA RUA" NA CIDADE DE TERESINA-PI
}

Profile of people in situation of social fragility served by the consultant on the street in the city of Teresina-PI

Perfil de las personas en situación de fragilidad social servido por el consultor en la calle de la ciudad de Teresina-PI

Samya Yasmin Sousa Silva • Estudante de Graduação $7^{\circ}$ período do Curso de Bacharelado em Serviço Social na Universidade Federal do Piauí-UFPI • bolsista do PET-Integração da UFPI • E-mail: samyas7980@hotmail.com

Lucia Cristina dos Santos Rosa • Professora Titular do Departamento de Serviço Social e do Programa de Pós-Graduação em Políticas Públicas da Universidade Federal do Piauí • E-mail: luciacsrosa@gmail.com

Melissa de Carvalho Soares Lima • Assistente Social da Fundação Municipal de Saúde de Teresina e Secretaria de Assistência Social e Cidadania de Teresina-PI • E-mail: melissalima4@hotmail.com

Autora responsável pela correspondência:

Samya Yasmin Sousa Silva • E-mail: samyas7980@hotmail.com 


\section{RESUMO}

Introdução: O Consultório na Rua (CNARUA) é uma equipe da Atenção Básica destinada a atender de modo integral a população em situação de fragilidade social na cidade de Teresina-PI. Objetivo: Esta pesquisa teve como objetivos traçar um perfil desse segmento da capital, conhecer as suas necessidades de saúde, além de comparar as características desta população com a Pesquisa Nacional. Método: Foi realizado o levantamento do perfil populacional a partir de informações de 174 prontuários ativos no ano de 2018, posteriormente comparado estatisticamente com as características apresentadas na Pesquisa Nacional. Durante a análise, observou-se as seguintes variáveis e aspectos: sexo, idade, pessoa de referência, procedência/naturalidade, o que a levou a viver nas ruas e a necessidade de saúde explicitada. Resultados: A análise dos dados mostrou um perfil dos usuários do CNARUA-THE como uma população predominantemente masculina (74,1\%), prevaleceu a faixa de 30 a 59 anos (71,85\%), originária do estado do Piauí (57,5\%). As principais razões para viver nas ruas, se referem aos conflitos familiares $(17,81 \%)$, seguida do consumo de substâncias psicoativas (9,19\%). Todos os resultados foram semelhantes aos da Pesquisa Nacional. Conclusões: O estudo das variáveis e aspectos reforçou a heterogeneidade do segmento populacional e ampliou o significado do que é o viver na rua, ainda apresentou as distinções e semelhanças do perfil da população local nacional. Logo, verifica-se a necessidade de compreender as especificidades de pessoas em situação de vulnerabilidade social no sentido de atendê-las adequadamente, consonante às suas necessidades de saúde, que por vezes extrapolam ações interventivas no âmbito da área.

Palavras-Chave: Consultório na Rua, População em Situação de Rua, Pesquisa Nacional População em Situação de Rua.

\section{BSTRACT}

roduction: The Consultant on the Street (CNARUA) a Primary Care team designed to fully assist the population in a situation of social fragility in the city of Teresina-PI. ective: This research aimed to outline a profile this segment of the capital, to know their health needs, in addition to comparing the characteristics of this population with the National Survey. Methods: A survey of the population profile was carried out based on information from 174 active medical records in 2018, later statistically compared with the characteristics presented in the National Survey. During the analysis, the following variables and aspects were observed: sex, age, reference of person, origin/naturality, which led her to live on the streets, and the need for explicit health. Results: Data analysis showed a profile of users of CNARUA-THE as a predominantly male population $(74.1 \%)$, the range of 30 to 59 years old $(71.85 \%)$, originating in the state of Piauí $(57,5 \%)$. The main reasons for living on the streets, refer to family conflicts $(17.81 \%)$, followed by the consumption of psychoactive substances $(9.19 \%)$. All results were similar to those of the National Survey. Co fisions: The study of variables and aspects reinforced the heterogeneity of the population segment and expanded the meaning of living on the street; 
and presented the differences and similarities the profile population local and national. Thus, there is a need to understand the specificities of people in a situation of social vulnerability in order to meet them properly, in line with their health needs, which sometimes go beyond interventional actions in the area.

Keywords: The Consultant on the Street, Population in Street Situation, National Survey Population in Street Situation.

\section{RESUMEN}

Introducción: El Consultor en la Calle (CNARUA) es un equipo de Atención Básica destinado a atender de manera integral a la población en una situación de fragilidad social en la ciudad de Teresina-PI. Objetivo: Esta investigación tuvo como objetivo trazar un perfil de este segmento de la capital, conocer sus necesidades de salud, además de comparar las características de esta población con la Encuesta Nacional. Método: fue realizado un análisis del perfil poblacional con base en la información de 174 registros médicos activos en 2018, posteriormente comparado estadísticamente con las características presentadas en la Encuesta nacional. Durante el análisis, se observaron las siguientes variables y aspectos: sexo, edad, persona de referencia, origen/lugar de nacimiento, lo que la llevó a vivir en la calle y la necesidad de salud que quedó explícita. Resultados: El análisis de datos mostró un perfil de usuarios del CNARUA-THE como una población predominantemente masculina (74.1\%), prevaleció el rango de 30 a 59 años $(71.85 \%)$, originarios del estado del Piauí (57,5\%). Las principales razones para vivir en la calle se refieren a conflictos familiares $(17,81 \%)$, seguidas del consumo de sustancias psicoactivas $(9,19 \%)$. Todos los resultados fueron similares a los de la Encuesta Nacional. Conclusiones: El estudio de variables y aspectos reforzó la heterogeneidad del segmento poblacional y amplió el significado de lo que es vivir en la calle; y presentó diferencias y semejanzas del perfil de la población local y nacional. Pronto, se concluye que es necesario comprender las especificidades de las personas en una situación de vulnerabilidad social para atender a ellas adecuadamente, de acuerdo con sus necesidades de salud, que a veces van más allá de las acciones de intervención dentro del área.

as Clave: El Consultor en la Calle, Población en situación de calle, Investigación Nacional Población en situación de calle. 


\section{ciência
pural}

\section{Introdução}

O “Consultório na Rua” (CNARUA) de Teresina surge inicialmente a partir do projeto "A arte de cuidar na rua", iniciando suas atividades em 15 de dezembro de 2010, vinculado inicialmente à Coordenação de Saúde Mental/Gerência de Ações Programáticas, da Fundação Municipal de Saúde do Município, sendo em 2012 transferido para a gestão da atenção básica. Como resposta ao edital lançado pelo governo federal e para responder ao contingente exorbitante de pessoas em situação de rua na Capital, observou-se a necessidade no âmbito da produção do cuidado, a partir de ofertas dos serviços de saúde para este segmento.

Foi constituído desde a origem por um corpo profissional multidisciplinar e multiprofissional, composto por: redutor de danos, enfermeira, assistente social e motorista, que atua apenas no turno da tarde, havendo apenas uma equipe para atender toda Teresina. Prestava atendimento clínico comunitário, voltado aos usuários de álcool, crack e outras drogas; em seus próprios contextos de vida na Capital. Inicialmente seus principais pontos de referência eram: Centro-Pop, Albergue e Praça da Bandeira.

Em 2017, o Consultório de Rua foi transferido da sede da Fundação Municipal de Saúde para o mesmo prédio do Centro de Referência Especializado de Assistência Social, na zona sul da Capital, fator positivo na articulação das ações dos diversos atores envolvidos na Política de atendimento a essa população. A partir daí, o atendimento da população em situação de rua, vinculou-se ao Departamento da Atenção Básica, Ministério da Saúde. Por esta razão, alterou a nomenclatura "Consultório de Rua" para "Consultório na Rua - CNARUA".

Dessa forma, entende-se que a inserção das equipes de saúde voltadas à população de rua no escopo das ações da atenção básica representa uma ampliação no sentido de efetivar os direitos à saúde desta população ${ }^{1}$. No mesmo ano, ocorreu a mudança de sede, passando a ser Unidade Básica de Saúde-UBS José Ribeiro de ho, situada no bairro Matinha. Os escopos de suas ações primam-se no 
cuidado integral, diante dos diversos problemas e necessidades de saúde da população em situação de rua.

Contornados pelo estigma que historicamente acompanhou esse segmento, discriminado geralmente por apresentar-se sem documentação e sem residência fixa, ter a higiene precária e muitas vezes transtorno mental, intermediado comumente pelo consumo de substâncias psicoativas. A equipe, ao ir de encontro a população usuária, adentra em um universo de valores e estratégias de sobrevivência que subverte a forma hegemônica de ser e estar no mundo, tornando-se uma das interlocutoras das necessidades e dos direitos desse segmento, mediante as políticas sociais e a sociedade em geral ${ }^{2}$.

Observando-se que as ações da equipe eram concentradas na ação direta com os usuários e na intermediação de seus direitos frente às políticas sociais, sobretudo no que diz respeito ao direito à saúde, principalmente cirurgias, cuidados pósoperatórios e ações de promoção em saúde, tais como teste rápido, ações educativas, distribuição de preservativos, tratamento diretamente observado em relação à tuberculose, havia parco conhecimento sobre o perfil geral dessa população no contexto local. A partir do estágio, buscou-se contribuir na construção de um perfil, haja vista ações do Serviço social na saúde que englobam em sua atuação ações de investigação, planejamento e gestão, conforme prevê os Parâmetros para Atuação de Assistentes sociais na Política de Saúde, que inclui a ação de realizar estudos e investigações com relação aos determinantes sociais da saúde ${ }^{3}$.

O Serviço Social se realça no atendimento em saúde a esse grupo de pessoas, devido a identificação dos determinantes sociais que restringem à condição existencial de vulnerabilidade da pessoa que não possui domicílio. Além disso, detém a compreensão da dimensão múltipla do usuário, levando em consideração os fatores sociais e econômicos que interferem no processo saúde-doença e sinaliza para que sua intervenção vá interferir, substancialmente, na necessidade de saúde deste 
Frente ao exposto, levantou-se a seguinte questão: quem são estes usuários que transitam no território, acompanhados pela equipe do CNARUA-THE? Quais as principais características destes usuários comparando-se com a Pesquisa Nacional e finalmente o que há em comum e divergente, entre o perfil da população de rua local versus nacional?

Na busca de conhecê-los, foi elaborado pela estagiária, supervisora de campo e docente vinculada a Universidade Federal do Piauí- UFPI, um perfil dos usuários atendidos pela equipe do CNARUA. Considerando-se a ampliação da resolutividade e longitudinalidade da produção do cuidado; na prevenção e promoção de saúde; este conhecimento é estratégico para a construção de linhas de cuidado, estabelecimento de prioridades, assim como melhor articulação com a rede de serviços.

Portanto, este trabalho teve como objetivos traçar um perfil da população em vulnerabilidade social na cidade de Teresina-PI, atendidos pelo CNARUA, comparar as características desta população com a Pesquisa Nacional, decifrando a realidade desse segmento populacional no referido município.

\section{Método}

A pesquisa censitária foi estruturada com base em 174 prontuários ativos no ano de 2018, cujas informações foram levantadas, mensuradas e registradas em uma matriz que destacou como principais variáveis: sexo, idade, procedência, naturalidade, estado civil, além de diversos aspectos como: motivo de estar na rua ou o que o levou a viver nas ruas, pessoa de referência, problemas/necessidade de saúde explicitada, forma de acesso ao serviço (demanda espontânea x encaminhada).

A análise do perfil das pessoas participantes da pesquisa foi posteriormente comparado pela estatística descritiva, com as características da população em situaçăo semelhante cadastradas na Pesquisa Nacional. Complementarmente, foi 
realizada revisão de literatura, que possibilitou interpretar as informações levantadas e intensificar a dimensão reflexiva.

\section{Resultados e Discussão}

O levantamento do perfil dos usuários do CNARUA-THE indicou uma população predominantemente masculina $(74,1 \%)$ e as pessoas do sexo feminino corresponderam a $(25,9 \%)$. Tal dado converge com os resultados obtidos na pesquisa “I Censo Nacional da População em Situação de Rua- 2007/2008” , que informa que a população em situação de rua é predominantemente masculina, com $82 \%$ de representação. Ressalta-se que foi identificado um prontuário de pessoa vinculada ao segmento LGBTI+, o que requer um repensar para a particularidade desse segmento.

Com relação à idade, distribuiu-se essa variável em 3 faixas, de 18 a 29 anos (segmento jovem); de 30 a 59 anos (adulta) e 60 anos e mais (população idosa). Prevaleceu a faixa de 30 a 59 anos, equivalente a 71,85\%, seguidamente, a faixa de 18 a 29 anos com $21 \%$ e de 60 ou mais que correspondeu a 4,02\%. Importante ressaltar que a pesquisa “I Censo Nacional da População em Situação de Rua-2007/2008”5 aponta para predominância da população adulta 53\%, dados que coincidem com a presente pesquisa.

No levantamento do perfil dos usuários do CNARUA-THE observou-se o número expressivo da população adulta, que está migrando para a faixa do envelhecimento, requerendo atenção para as enfermidades crônicas degenerativas, como hipertensão e diabetes. Com relação às pessoas de referência, o levantamento do perfil dos usuários do CNARUA- THE constata que em torno de 36\% dos usuários possuem alguma pessoa de referência, sobretudo, do sexo feminino, destacando-se a mãe, irmã ou ex-esposa. Esse percentual mostrou-se inferior a Pesquisa Nacional ${ }^{5}$ que evidenciou um percentual de 51,9\% dos entrevistados com vínculos familiares.

No que se refere à procedência, o levantamento do perfil dos usuários do UA-THE revelou que a maior taxa da população em situação de rua é 
originária do estado do Piauí (57,5\%); parte significativa corresponde ao Maranhão $(17,2 \%)$ e outros estados somaram 15,5\%. Esses resultados foram reiterados pelos apresentados na Pesquisa Nacional ${ }^{5}$, onde $45,8 \%$ dos entrevistados sempre viveram no município em que moram atualmente. Do restante $(54,2 \%)$ do total, $56 \%$ vieram de municípios do mesmo estado de moradia atual e $72 \%$ vieram de áreas urbanas.

Dos entrevistados na presente pesquisa, a maioria advém do mesmo local da sede do consultório, ou seja, Teresina, ou de municípios próximos, tais como Campo Maior, Altos, Timon-MA. Tal informação sinaliza para maior possibilidade da equipe do CNARUA poder acessar famílias/familiares, ou atuar com base nas experiências dessas pessoas com a própria cidade de Teresina, o que permite maior diálogo com a rede de sociabilidade primária, comunitária, na perspectiva de construção de outras possibilidades para este segmento. Por fim, as principais razões para viver nas ruas, se referem aos conflitos familiares (17,81\%). Em seguida aparece o consumo de substâncias psicoativas (9,19\%). De forma semelhante, o "I Censo Nacional da População em Situação de Rua- 2007/20085 verificou que um dos principais motivos que levam a pessoa a morar na rua refere-se ao alcoolismo e uso de drogas (35,5\%).

De acordo com o levantamento do perfil dos usuários desta pesquisa o álcool $(23,5 \%)$ aparece como destaque, mas comumente é misturado com outras substâncias psicoativas em torno de $15 \%$. Em contrapartida, o consumo de crack $(23,5 \%)$ é misturado com outras substâncias psicoativas em torno de $(7,47 \%)$, configurando consumo cruzado. Aliás, o consumo de substâncias psicoativas é um fato corriqueiro no ambiente das ruas que acaba funcionando como elemento socializador, fonte de prazer, automedicação ou meio de conseguir alguma renda, que de certa forma também contribui para o agravo dos inevitáveis problemas tanto físicos quanto mentais ${ }^{6}$.

Nota-se que o consumo de álcool está em quantidade elevada, em proporções hantes ao crack. Em vista disto, é importante considerar as mudanças do e das políticas públicas sobre drogas no Brasil, de um discurso pensado para 
uma "sociedade livre de drogas". Em função da realidade observada em diversos locais do país, este deve ser substituído pelo discurso ideal de uma "sociedade protegida do uso de drogas ilícitas e do uso indevido de drogas lícitas"7. As principais causas apontadas sobre os problemas encontrados são relacionados a fatores estruturais como desemprego e falta de condições financeiras $(5,74 \%)^{7}$.

As três primeiras razões que apareceram na pesquisa "I Censo Nacional da População em Situação de Rua 2007/2008"5, apresenta os principais motivos pelos quais essas pessoas passaram a viver e morar na rua, os quais se referem aos problemas de alcoolismo e/ou drogas (35,5\%), desemprego $(29,8 \%)$ e desavenças com pai/mãe/irmãos (29,1\%). Observa-se que, os principais motivos citados estão correlacionados, ao consumo de substâncias psicoativas, acompanhado de conflitos familiares.

Na presente pesquisa verifica-se um motivo a mais, a morte de algum dos genitores ou ambos $(8,62 \%)$, demonstrando que o processo do luto desestrutura psicologicamente o indivíduo, a ponto de levá-lo a situação de rua. Verificou-se que majoritariamente as razões que levaram as pessoas a situações de rua estão associadas a conflitos ou experiências negativas, como perdas por morte ou separações. Este motivo não foi constatado na Pesquisa Nacional ${ }^{5}$.

Identificou-se o registro de alguns outros motivadores considerados distintos, representados por 4,02\%, evidenciando-se: curiosidade, acompanhando companheiro/ou mãe e prazer/liberdade. Há também a representação, embora minoritária, de pessoas que tem um estilo de vida nômade, ou seja, que vive mudando de lugar 8 , tais como os trabalhadores de circo e ciganos.

Logo, apesar do sofrimento e/ou conflitos estarem representados, há pessoas que se encontram em situação de rua por opção. Há ainda, pessoas que estão foragidas da justiça ou com pendência/litígios judiciais, que persistem em morar na rua como estratégia de sobrevivência. 
Dentre as necessidades de saúde mais citadas destacam-se: consumo de substâncias psicoativas $(15,55 \%)$, sífilis $(9,77 \%)$, tratamento odontológico $(9,19 \%)$, transtorno mental (7,47 \%); e tuberculose (4,02 \%). O I Censo Nacional da População em Situação de Rua-2007/20085 mostra que entre os problemas de saúde mais citados destacam-se: hipertensão (10,1\%), problema psiquiátrico/mental (6,1\%), HIV/Aids $(5,1 \%)$ e problemas de visão/cegueira $(4,6 \%)$.

O transtorno mental tem maior incidência igualmente em ambas as análises. Geralmente esse tipo de transtorno antecede à condição de se morar nas ruas. A condição precária de viver nas ruas e o consumo de substâncias psicoativas podem exacerbar os seus sintomas anteriores, assim como favorecer o aparecimento de outros transtornos, levando a uma alta prevalência de comorbidade nesses indivíduos, tornando os casos ainda mais graves 9 .

No I Censo Nacional da População em Situação de Rua- 2007/20085 mostra-se mais prevalente o HIV/Aids do que no levantamento do perfil dos usuários do CNARUA-THE. Conforme uma pesquisa atual, a exposição ao HIV à pessoa em situação de rua decorre principalmente da relação entre maior/menor frequência de uso do preservativo, tipo de parceria sexual e consumo de substâncias psicoativas; um fator que dificulta a decisão do indivíduo em utilizar o preservativo, especialmente quando o uso ocorre antes das relações sexuais ${ }^{10}$.

No levantamento do perfil dos usuários do CNARUA-THE, os dados revelam: a incidência de Infecções Sexualmente Transmissíveis-IST é elevada nesse grupo, o que revela as dificuldades no manejo de ações preventivas ou sexo protegido no contexto da rua. A sexualidade é uma temática muito viva nesse grupo social11.

Cabe ressaltar ainda, que a tuberculose se configura hoje, uma emergência epidemiológica dos usuários do Consultório na Rua em Teresina. As condições de exclusão social que eles vivem, torna-se um risco de desenvolver a doença ou tê-la da por dificuldades de dar continuidade ao tratamento, até pelos efeitos ais adversos, o que torna difícil a adesão, em função do tempo exigido para 
sua eficácia, feito sob monitoramento da equipe do CNARUA-THE. Sendo assim, é perceptível uma pulverização de problemas vinculados à saúde, muitos deles, conjugados. Portanto, apreende-se que infelizmente as adversas situações que permeiam a vida nas ruas acabam por criarem situações de riscos à saúde física e mental ${ }^{12}$.

\section{Conclusões}

A partir das análises realizadas nesta pesquisa, apreende-se que a realidade da população em situação de rua na cidade de Teresina conversa com a do resto do país, apresentada na Pesquisa Nacional, apesar de algumas distinções elencadas. É importante, compreender a respeito desse segmento, composto por vários perfis de indivíduos que tem modo, cotidiano e trajetória de vida singular.

A análise das variáveis e aspectos mostrou a heterogeneidade desse grupo social e ampliou o significado do que é o viver na rua, trazendo à cena o que há em comum e divergente entre o perfil dessa população local versus nacional. Verifica-se a necessidade de compreender as especificidades de pessoas em situação de vulnerabilidade social no sentido de atendê-las de forma adequada, diante de suas necessidades de saúde.

Por fim, o conhecimento dos usuários assim como de seus problemas e necessidades é estratégico para produzir cuidado e melhor articular a rede socioassistencial. Além disso, auxilia aos trabalhadores da saúde, na coordenação do cuidado, na oferta de um atendimento humanizado, viabilizando o estabelecimento do vínculo, conjuntamente, favorecendo sua adesão ao tratamento.

\section{ferências}

1. Santana C. Consultórios de rua ou na rua? Reflexões sobre as políticas de abordagem à saúde da população de rua [carta]. Cad. Saúde Públic. 2014; 30(8):17981799.

2. Marques MD. A rua como espaço profissional do serviço social na saúde: a do assistente social no consultório na rua de Teresina. Capítulo XXII. In: 
Pereira SLB (Org.). O processo de trabalho do serviço social na saúde mental. 1. ed. Teresina: EDUFPI, 2017. 260p.

3. Conselho Federal de Serviço Social. Parâmetros para atuação de assistentes sociais na política de saúde. 2010; 1(1): 82-10.

4. Martins SSS, Silva EM. Análise da condição de saúde bucal da população em situação de rua do município de Natal-RN. Revista Ciência Plural.2019; 5(3):21-39.

5. Ministério do Desenvolvimento Social e Combate à Fome[internet]. Pesquisa nacional sobre a população em situação de rua. [atualizado em 16 abr 2008; citado em 20 out 2018]. Disponível em: http://www.mds.gov.br/backup/arquivos/sumario_executivo_pop_rua.pdf .

6. Rosa S, Santana L. Consultório na Rua como boa prática em Saúde Coletiva. Rev. Bras. Enferm. 2018;71(1):501-502. http://dx.doi.org/10.1590/0034-7167201871sup102.

7. Conselho Nacional de Política sobre Drogas. Resolução $n^{0} 01$, de 6 de maio de 2015. Regulamento para entidades que realizam o acolhimento de pessoas, em caráter voluntário, com problemas associados ao uso nocivo ou dependência de substância psicoativa. Sistema Nacional de Políticas Públicas sobre Drogas 7 mai 2015; Seção 1.

8. Campos A. População de rua: um olhar da educação interprofissional para os não visíveis. Saude soc. 2018; 27(4):997-1003. https://doi.org/10.1590/S010412902018180908

9. Lovisi GM, Lima LA, Morgado AF. População de rua e os transtornos mentais maiores: revisão dos aspectos relevantes. Jornal Brasileiro de Psiquiatria. 2001 jan; 5(6):149-160.

10. Grangeiro A, Holcman MM, Onaga ET, Alencar HDR de, Placco ALN, Teixeira PR. Prevalência e vulnerabilidade à infecção pelo HIV de moradores de rua em São Paulo, SP. Rev. Saúde Pública. 2012 jun; 46(4): 674-684.

11. Brasil. Ministério da Saúde. Secretaria de Atenção à Saúde. Departamento de Atenção Básica. Manual sobre o cuidado à saúde junto à população em situação de rua. Brasília: Ministério da Saúde; 2012.

12. Silva F, Silva El, Ferreira A, Frazão I. Segurança do paciente: ao alcance das pessoas em situação de rua? Rev. Bras. Enferm. 2020;73(5):01-04. https://doi.org/10.1590/0034-7167-2019-0114. 